\title{
Le transfert d'un jeu de rôles, de la recherche scientifique à la stratégie d'éducation
}

\section{Frédérique Chlous-Ducharme}

\author{
(2) OpenEdition \\ Journals \\ Édition électronique \\ URL : http://journals.openedition.org/ere/1632 \\ DOI : $10.4000 /$ ere. 1632 \\ ISSN : 2561-2271 \\ Éditeur \\ Centr'ERE
}

Référence électronique

Frédérique Chlous-Ducharme, "Le transfert d'un jeu de rôles, de la recherche scientifique à la stratégie d'éducation », Éducation relative à l'environnement [En ligne], Volume 9 | 2011, mis en ligne le 20 décembre 2011, consulté le 21 février 2020. URL : http://journals.openedition.org/ere/1632 ; DOI 10.4000/ere.1632

Ce document a été généré automatiquement le 21 février 2020. 


\title{
Le transfert d'un jeu de rôles, de la recherche scientifique à la stratégie d'éducation
}

\author{
Frédérique Chlous-Ducharme
}

1 Une double préoccupation s'impose aujourd'hui dans la gestion des espaces qualifiés de naturels : le développement durable et, conjointement, la participation des acteurs à l'exercice du politique. Le champ de l'environnement soulève des problèmes complexes entrainant conflits et controverses, et l'élaboration des propositions de gestion passe en partie par la mobilisation d'acteurs hétérogènes. Si le choix politique et sociétal est celui du développement durable et de l'association des citoyens à la gestion des affaires publiques, il est important de réfléchir à des stratégies pédagogiques permettant le partage de connaissances, et la construction participative de projets de société. Les enjeux que posent les interactions entre dynamiques sociales et dynamiques naturelles peuvent être abordés dans le cadre d'un jeu de rôles. La discussion proposée dans ce texte a trait à l'application, en milieu scolaire, d'un jeu de rôles portant sur l'aménagement d'un milieu de vie. Ce jeu de rôle a d'abord été développé pour la communauté de l'île d'Ouessant, qui a vu son mode de vie et son paysage changer considérablement depuis les soixante dernières années. Il a ensuite été adapté pour être réalisé avec des élèves de lycée en visite à Océanopolis, un parc de découverte des océans situé à Brest. Le jeu vise à ce que, dans un climat ludique, les participants s'intéressent aux processus environnementaux se déroulant dans le temps et l'espace, et acquièrent des compétences pour prendre part aux discussions portant sur les modalités de gestion d'un territoire.

2 L'île d'Ouessant (département du Finistère, France) fait partie du Parc naturel régional d'Armorique et de la Réserve de biosphère de la mer d'Iroise. Dans les soixante dernières années, on y a remarqué une diminution et un vieillissement de la population. L'augmentation massive du nombre de résidences secondaires, le quasiabandon des activités agricoles traditionnelles et l'accroissement considérable du tourisme en ont modifié les paysages. L'embroussaillement de l'île témoigne ainsi d'un 
monde qui n'est plus, d'un délaissement des pratiques traditionnelles. Les Ouessantins le mettent en parallèle avec l'affaiblissement des solidarités. Bien que la population locale déplore le non-entretien des terres, elle intervient peu pour contenir l'embroussaillement. En effet, si le pourtour des maisons et certaines prairies sont "nettoyés ", les espaces non utilisés - ou non visibles depuis les habitations - sont peu défrichés par les particuliers. Seuls les sites classés, gérés par le Parc naturel régional d'Armorique, sont régulièrement débroussaillés, ainsi que les pourtours des routes nettoyés par les employés communaux. Les moyens de ces deux institutions ne permettent pas aujourd'hui de défricher davantage.

Pratiquement inexistantes dans les années 1950, les friches se sont développées depuis, occupant plus de la moitié du territoire (Gourmelon et al., 1995). Différents enjeux notamment paysagers, écologiques et culturels - sont à considérer si l'on souhaite planifier l'aménagement des terres de l'île suite à leur changement d'utilisation. Si de nouveaux résidents rejoignent les discours des Ouessantins d'origine, considérant comme regrettables la fermeture des espaces autrefois cultivés et la disparition d'un paysage modelé par les activités traditionnelles, d'autres apprécient au contraire les friches qui les prémunissent des regards inquisiteurs et des visiteurs inopportuns (Chlous-Ducharme et al., 2008). Certains habitants veulent garder des vues sur la mer, d'autres encore ont le souci de favoriser la biodiversité et de protéger les milieux naturels, plusieurs souhaitent que le paysage témoigne des modes de vie du passé.

\section{Conséquences des évolutions démographiques et des modes de vie sur les pratiques ouessantines}

4 À côté de l'entretien mécanique des espaces, le pâturage des moutons a longtemps participé au maintien des milieux. Les moutons, beaucoup moins nombreux que dans le passé et appartenant à des résidents de plus en plus âgés, sont désormais considérés comme des animaux de compagnie. L'évolution de la population et la transformation des modes de vie sur l'île d'Ouessant ont également des incidences sur l'étrépage. Cette activité consiste à prélever sur la frange littorale des mottes ${ }^{1}$ de terre qui serviront de combustible. Elle se pratique aujourd'hui beaucoup moins et ne se réalise plus dans le même but qu'autrefois. Cette pratique, banale pour chaque famille dans le passé, s'est déplacée de la sphère domestique à la sphère commerciale. Si quelques Ouessantins pratiquent encore l'étrépage pour leurs besoins personnels, des étrépeurs "professionnels» ramassent les mottes sur quelques parcelles, facilement accessibles en voiture, pour permettre aux restaurateurs de proposer aux touristes - très friands de consommer « local»- de goûter à la spécialité ouessantine : le ragoût sous la motte ou cuisson buaden (Joets, 2005). Une dégradation de certaines zones littorales est alors visible, et se pose la question de la gestion de cette pratique qui doit concilier la conservation d'un patrimoine naturel et la pérennité d'un patrimoine culturel. Le dérangement par la fréquentation touristique importante sur les chemins côtiers et l'étrépage affectent les conditions de survie du crave à bec rouge. Cet oiseau, qui niche dans les falaises d'Ouessant, présente un intérêt patrimonial et a conduit à la mise en place de Zones de protection spéciales (ZPS, directive européenne). 


\section{La construction d'un jeu de rôles}

5 Le jeu de rôles a été construit par une équipe multidisciplinaire de scientifiques. L'élaboration de ce jeu s'est appuyée sur les travaux du collectif ComMod $(2005,2006)$, spécialisé en modélisation. La démarche de conception a d'abord reposé sur l'utilisation d'une technique de modélisation, soit le système multiagents (SMA) ${ }^{2}$. Le SMA a ensuite été utilisé pour la réalisation d'un prototype informatique, qui a permis d'observer les évolutions possibles du milieu ouessantin sur une durée de vingt ans. À la suite de ces travaux (Gourmelon et al., 2008), l'équipe pluridisciplinaire s'est orientée vers la création du jeu de rôles, dont les objectifs principaux étaient de sensibiliser les habitants d'Ouessant et les collectivités territoriales aux questions de l'embroussaillement et de la dégradation de la frange littorale et de renforcer la capacité des acteurs locaux à intervenir dans les procédures de gestion. Il a été choisi de construire un jeu réaliste, mais non figuratif, en représentant la plupart des écosystèmes que l'on retrouve à Ouessant: la mer, une bande côtière de pelouse littorale, des agrégats de lande littorale, des zones de broussailles hétérogènes, une surface importante de prairie, une route goudronnée, un chemin en terre, et des maisons disposées par les joueurs.

6 Le jeu favorise les interactions entre les participants et leur permet de tester ou d'inventer des solutions de gestion. Neuf joueurs, représentant différents types d'acteurs, interviennent: résidents principaux et natifs du territoire, résidents principaux arrivés récemment, résidents secondaires, élus, porte-parole du Parc naturel régional d'Armorique. Chacun de ces acteurs peut agir dans le jeu en intervenant sur l'élevage de moutons ou de vaches, l'étrépage, la fauche, la commande de mottes pour la restauration, la mise en place de cultures cynégétiques. À chaque tour de jeu, qui représente une période de deux ans, l'état de la végétation est réactualisé ${ }^{3}$ en fonction des actions des différents joueurs. Dix tours constituent une partie et permettent ainsi de saisir l'évolution du territoire sur 20 ans.

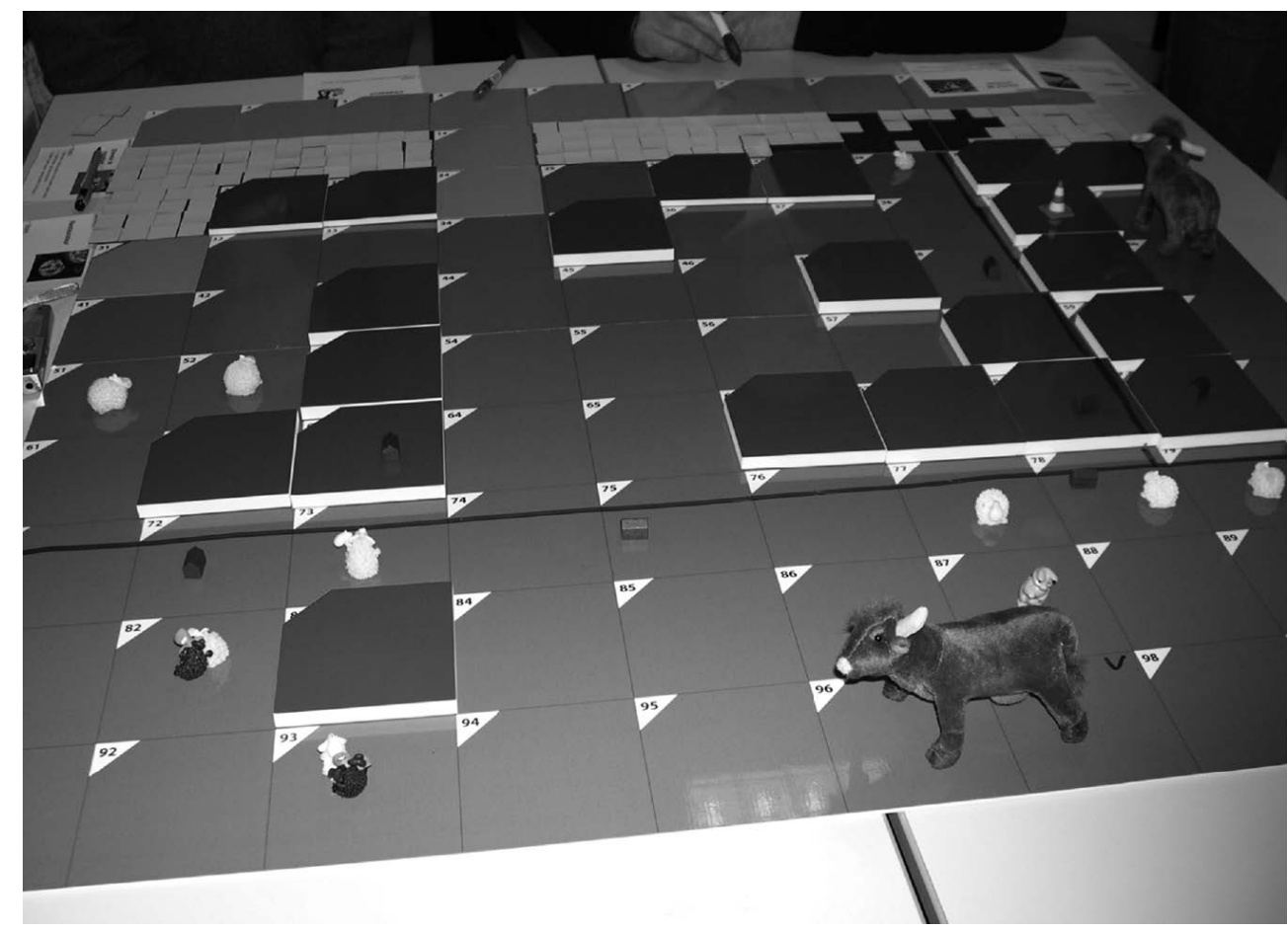

Éducation relative à l'environnement, Volume 9 | 2011 
Figure 1 : Le plateau de jeu photographié lors d'une partie

7 En cours d'élaboration du jeu, nous avons fait appel à Océanopolis, un organisme qui possède une solide expérience concernant la réalisation d'ateliers auprès des scolaires et du grand public. Nous avons discuté avec eux de l'utilité d'un tel matériel pédagogique et de ses possibilités d'adaptation, tant sur le fond que sur la forme. Ce partenariat a débouché sur une opportunité de réalisation du jeu au sein même du parc de découverte, et ce, auprès d'une clientèle scolaire.

Le personnel du service éducatif d'Océanopolis et des enseignantes conseillères-relais du programme Art et culture du ministère de l'Éducation nationale ont analysé la pertinence du jeu de rôles en fonction des missions qui leur sont attribuées. Une analyse fine des programmes élaborés par le ministère de l'Éducation nationale a montré que le matériel proposé présentait un intérêt pédagogique pour les élèves de troisième et de seconde et touchait plusieurs thématiques: évolution de l'environnement (considéré comme l'espace aménagé par les sociétés humaines), rôle de l'humain et de la société dans l'aménagement du territoire, impact des activités humaines sur les espaces littoraux, protection et gestion de l'environnement, fragilité des écosystèmes. Les lycéens ont ainsi été choisis comme public cible de cette adaptation du jeu de rôles. Le jeu a finalement été intégré en 2009 à un atelier thématique offert par Océanopolis intitulé «Entre terre et mer, les littoraux». Cet atelier comporte également une visite commentée du pavillon tempéré d'Océanopolis et la réalisation d'un croquis de synthèse sur les enjeux de gestion de la mer d'Iroise.

9 Après la présentation des objectifs de l'utilisation du jeu de rôles auprès d'un public scolaire, nous discuterons de son potentiel quant à l'acquisition de connaissances et de compétences.

\section{Les objectifs de réalisation du jeu auprès d'un public scolaire}

10 Les objectifs formulés par l'équipe de chercheurs et de partenaires à l'origine de la version scolaire du jeu concernent l'acquisition de savoirs naturalistes (développement de la friche, restauration des milieux, action du pâturage, impacts des activités humaines) et de connaissances en sciences humaines et sociales (différenciations sociales des pratiques et systèmes de représentation). Tous ont aussi insisté sur la nécessité de croiser ces deux champs de savoirs et de favoriser l'acquisition de savoirfaire relatifs à la mise en mot d'un problème, à la compréhension des codes pour participer à la discussion, à l'écoute de l'autre et à l'appréhension des différences de point de vue. Le jeu vise à former, sous un mode ludique, de futurs citoyens à la discussion sur la gestion des territoires par l'appropriation de savoirs et de savoir-faire. Il entend permettre de sensibiliser les lycéens aux processus environnementaux et sociaux se déroulant à de multiples échelles, et de contribuer à l'expérimentation in ludo des principes et contraintes de la participation citoyenne. 


\section{Discussion}

La réalisation du jeu en 2009 et 2010 auprès de différentes classes de troisième et de seconde a permis une première analyse. Plusieurs classes venues de toute la France se sont inscrites dans l'atelier "Entre terre et mer, les littoraux», proposé par Océanopolis. Trente-trois séances de jeu ont ainsi été mises en œuvre et quinze d'entre elles ont été observées par les chercheurs porteurs du projet initial et par le personnel d'Océanopolis, suivant un guide d'observation préalablement construit. L'aspect ludique, les règles simples et le rassemblement autour d'un plateau de jeu stimulent les interactions. L'embroussaillement inexorable du «territoire » conduit les participants, après quelques tours de jeu, à discuter entre eux et à prendre des initiatives communes à l'ensemble du groupe ou à quelques joueurs. Par exemple, les joueurs tentent de contenir les friches dans certains secteurs en ayant plutôt tendance à privilégier la côte, les alentours des maisons, ou expérimentent l'utilisation de nouveaux moyens (achat de machines, développement de l'agriculture, augmentation du nombre de moutons, ou encore constitution d'un troupeau de chèvres pour débroussailler). Il a été plus difficile d'établir si les interactions entre les dynamiques sociales et naturelles ont été comprises et analysées. Des études complémentaires doivent être réalisées en ce sens.

L'approche privilégiée a visé à ce que les participants puissent acquérir des savoirs et savoir-faire tout en construisant une réflexion critique autonome. Il a donc importé de ne pas imposer de "bonnes manières de jouer ", que ce soit au sujet de résultats sur le plateau de jeu/territoire ou de la qualité de la discussion. Aussi, jouer à un jeu signifie, pour les élèves, qu'à la fin de la partie il soit possible d'identifier des gagnants et des perdants. Or, cela ne peut pas être le cas avec le jeu de rôles présenté. Comment alors éviter que le contrôle ou la réduction des friches, qui produisent un territoire "beau " et "propre", soient associés au fait de gagner? Comment susciter la discussion à propos d'autres critères, notamment écologiques ${ }^{4}$ ? Ensuite, que dire de la qualité de la discussion lors du jeu ? Y a-t-il une bonne conduite basée sur une discussion ouverte à tous, sans conflits, et construite autour d'arguments raisonnés? Tous les arguments peuvent-ils être entendus? Enfin, comment mettre en discussion le concept de développement durable et ne pas l'imposer? Cette posture semble d'autant plus complexe à tenir que le modèle de développement durable est largement partagé par l'ensemble de la population, et que le système éducatif français a mis en œuvre une large opération de sensibilisation à ce concept. Alors que ce jeu a moins d'une année d'existence, les questions posées interrogent les conditions du transfert d'un outil construit dans le cadre d'une recherche scientifique menée sur un territoire concret avec les acteurs concernés vers une stratégie d'éducation de lycéens. Une première analyse a montré que le jeu permet aux élèves de se saisir de la problématique d'une gestion concertée d'un territoire, mais les questions épistémologiques et éthiques restent en discussion.

13 L'expérience a fait ressortir que des liens étroits doivent se construire entre les chercheurs et les professionnels qui mettent en œuvre les séances de jeu. Les objectifs doivent être clairement explicités et partagés même si les connaissances et compétences sont réparties de manière différenciée entre les chercheurs de différentes disciplines, les responsables et les animateurs de la structure Océanopolis, ainsi que les conseillères-relais du ministère de l'Éducation nationale. 


\section{BIBLIOGRAPHIE}

Chlous-Ducharme, F. et Bellec, S. (2008). Les résidents secondaires à Ouessant : acteurs de la fabrication des paysages. Dans Philippe Jarnoux (dir.) La lande. Un paysage au gré des hommes (p. 189-200). Le Faou : CRBC/PNRA.

Collectif ComMod (2005). La modélisation comme outil d'accompagnement. Natures Sciences Sociétés, 13/2, 165-168.

Collectif ComMod (2006). Modélisation d'accompagnement. Dans Amblard, F. et Phan, D. (eds). Modélisation et simulation multi-agent : applications aux sciences de l'homme et de la société (p. 217-228). Londres : Hermes Sciences.

Gourmelon, F., Bioret, F. et Brigand, L. (1995). SIG et usage des sols : l'île d'Ouessant de 1952 à 1992. Mappemonde, 4, 6-10.

Gourmelon, F., Etienne, M., Rouan, M., Kerbiriou, C., Charles, M., Bioret, F., Chlous-Ducharme, F., Guermeur, Y. et Hevrel, H. (2008). Éléments de prospective environnementale dans une réserve de biosphère. Cybergeo, Systèmes, Modélisation, Géostatistiques, article 429, mis en ligne le 25 septembre 2008, modifié le 06 octobre 2008. URL : http://www.cybergeo.eu/index20343.html.

Joets, A. (2005). Le ramassage des mottes à Ouessant. Enjeu et valorisation d'un patrimoine culturel et naturel. Mémoire Master 1 Culture et société. Université de Bretagne Occidentale.

\section{NOTES}

1. La motte est constituée par la couverture végétale et la terre qui l'a nourrie. L'épaisseur de sol enlevée est de 5 à $10 \mathrm{~cm}$.

2. Un SMA est composé d'agents, humains ou non humains (ex. : troupeau de moutons), qui interagissent dans un environnement donné.

3. Un modèle informatique est couplé au jeu et permet de calculer le changement d'état des différents types de végétation.

4. En effet, si l'on excepte la population de crave à bec rouge, les connaissances scientifiques actuelles ne démontrent pas une perte de la biodiversité suite à l'embroussaillement, mais plutôt une recomposition de celle-ci.

\section{AUTEUR}

\section{FRÉDÉRIQUE CHLOUS-DUCHARME}

Maître de conférences - Habilitée à Diriger des Recherches - à l'Institut de géoarchitecture et membre du laboratoire « Conception, aménagement et gestion du cadre bâti et de l'environnement. Doctrines et pratiques » de l'Université de Bretagne Occidentale. Ses recherches portent principalement sur les modalités de gestion des espaces qualifiés de naturels. 